\title{
Movements of molecular motors: Ratchets, random walks and traffic phenomena Stefan Klumpp ${ }^{\mathrm{a}, 1}$, Theo M. Nieuwenhuizen ${ }^{\mathrm{b}}$ and Reinhard Lipowsky ${ }^{\mathrm{a}}$ \\ ${ }^{\mathrm{a}}$ Max-Planck-Institut für Kolloid- und Grenzflächenforschung, 14424 Potsdam-Golm, Germany \\ ${ }^{\mathrm{b}}$ Instituut voor Theoretische Fysica, Universiteit van Amsterdam, Valckenierstraat 65, 1018 XE Amsterdam, The Netherlands
}

\begin{abstract}
Processive molecular motors which drive the traffic of organelles in cells move in a directed way along cytoskeletal filaments. On large time scales, they perform motor walks, i.e., peculiar random walks which arise from the repeated unbinding from and rebinding to filaments. Unbound motors perform Brownian motion in the surrounding fluid. In addition, the traffic of molecular motors exhibits many cooperative phenomena. In particular, it faces similar problems as the traffic on streets such as the occurrence of traffic jams and the coordination of (two-way) traffic. These issues are studied here theoretically using lattice models.
\end{abstract}

Key words: molecular motors, active movement, random walks, lattice models, traffic jams PACS: 87.16.Nn, 05.40.-a, 05.60.-k

\section{Introduction}

The idea of constructing nanometer-sized devices and machines has created a lot of excitement during the last years. Despite the progress made, the functionality of artificial nano-devices is, however, still rather limited. At the same time, more and more biomolecular nano-machines have been identified in the cells of living beings where they accomplish a huge variety of tasks. Many of these molecular motors are now rather well studied and were found to work with an amazing degree of precision and efficiency as a result of billions of years of evolution, $[1,2]$. In the following, we will focus on one class of molecular motors which has

\footnotetext{
1 Corresponding author. E-mail: klumpp@mpikggolm.mpg.de
}

been studied quite extensively during the last decade, namely processive cytoskeletal motors which drive the traffic of vesicles and organelles within cells. These motors hydrolyze adenosinetriphosphate (ATP) and convert the free energy from this chemical reaction into directed movements along filaments of the cytoskeleton. This class of motors contains kinesins and dyneins, which move along microtubules, and certain myosins, which move along actin filaments. These motors walk along the filaments by performing discrete steps with a step size which corresponds to the repeat distance of the filament, $8 \mathrm{~nm}$ for kinesins and $36 \mathrm{~nm}$ for myosin $\mathrm{V}$. They are called processive if they make many steps while staying in contact with the filament.

From a physical point of view, much of the interest in molecular motors is due to the fact that the difference in size compared to macroscopic engines implies also 
conceptual differences. The typical energy of macroscopic motors is much larger than the thermal energy, $k_{\mathrm{B}} T$, while the typical energies of molecular motors are of the order of $k_{\mathrm{B}} T$. For example, the hydrolysis of ATP releases about $20 k_{\mathrm{B}} T$. On the one hand, molecular motors have to cope with perturbations arising from thermal fluctuations; on the other hand, the unavoidable presence of noise suggests that evolution has created motors which make use of this noise in order to generate work or directed movement.

From a technological viewpoint, the amazing properties of single biological motor molecules and the complexity of the systems into which they are integrated in the cell provide inspiration for the design of artificial nanoscale transport systems $[3,4]$.

In this article, we discuss several theoretical aspects of the motor movements. In section 2, we start by summarizing some recent experimental results and discuss the question whether noise-driven mechanisms are used by these motors. In section 3 , we discuss another effect of noise, namely the detachment of motors from their tracks due to thermal fluctuations, which leads to peculiar random walks. We derive the asymptotic behavior of these random walks using the statistical properties of the returns of motors to the filament. Finally, in section 4 , we summarize our recent studies of traffic problems which arise in systems with many molecular motors due to their mutual exclusion from binding sites of the filaments. These topics are also addressed in a recent longer review article [5].

\section{Active movements of molecular motors}

Molecular motors can be studied outside cells using biomimetic model systems. In these experiments, the biological complexity is reduced to a minimal number of components, namely motors, filaments, and ATP. An example is shown in Fig. 1. By these experiments, one can observe movements of single motor molecules and measure transport properties such as velocities, step sizes, and forces $[1,2]$.

On the one hand, these experiments provide insight into the motor mechanisms. A major breakthrough was to resolve the discrete steps of the motors and to measure the step size of the motor which corresponds to the repeat distance of the filament. This has first been

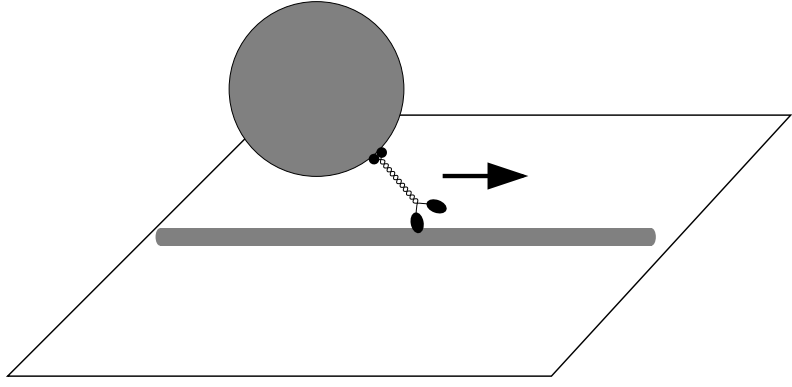

Fig. 1. The 'bead assay' constitutes a biomimetic model system: A molecular motor transports a (glass or latex) bead along a filament which is immobilized on a surface.

achieved for kinesin using an optical tweezers setup [6]. More recently, it has been shown that kinesin $[7,8]$ and also myosin $\mathrm{V}$ [9] move in a hand-over-hand way, i.e., that the two heads of the dimer step forward in an alternating fashion such that the rear head always moves in front of the leading head, similar to human walking. Further progress is expected from combining mechanical methods and particle tracking with fluorescence techniques.

On the other hand, using these biomimetic motility assays, one can measure the transport properties systematically varying external control parameters. Here the main focus has been on the velocity as a function of the ATP concentration and of the force applied with, e.g., optical tweezers to oppose the movements, see, e.g., Ref. [10]. Other quantities that have been measured are the one-dimensional diffusion coefficient of motors bound to filaments or the randomness parameter and the walking distance before unbinding from the filament. These measurements have stimulated a large amount of theoretical work, see, e.g. Refs. [11,12,13], modeling the walks of motors along filaments in order either to fit the experimental data or to find out the generic properties of these walks. For example, it turns out that the motor velocity as a function of the ATP concentration is given by a universal relationship which should be valid for many types of motors $[14,15]$.

Since nanometer-sized molecular motors have to live in a noisy environment, it has soon been speculated whether these motors exploit the noise to generate their directed movements, and various variants of ratchet models have been proposed as reviewed in Refs. $[11,12,13]$. In the simplest case, the conformational changes associated with the chemical cycle of the 


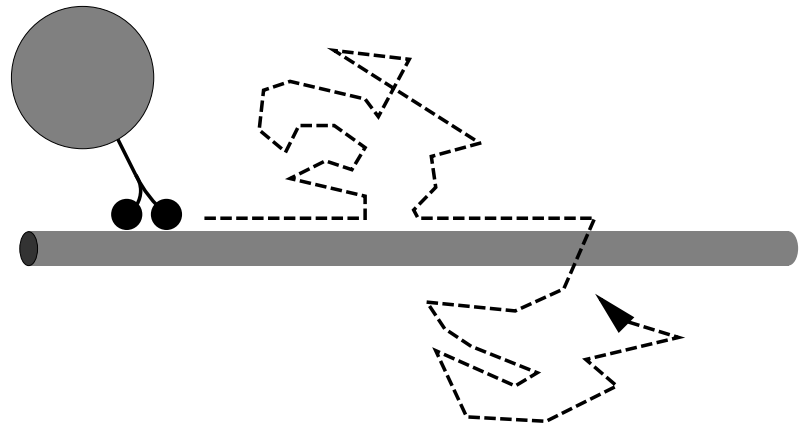

Fig. 2. Random walk of a molecular motor: The motor performs directed movement along a filament and unbinds from it after a certain walking distance. The unbound motor diffuses in the surrounding fluid until it rebinds to the filament and resumes directed motion.

motor rectify the one-dimensional Brownian motion along the filament. While such a simple mechanism is not consistent with the measurements for dimeric motors such as conventional kinesin or myosin $\mathrm{V}$, it can describe movements of processive monomeric motors such as the monomeric kinesin $\operatorname{KIF} 1 \mathrm{~A}[16,17,18]$ (for other processive monomeric motors, see the review [19]). These monomeric motors exhibit biased, but strongly diffusive movements along the filaments similar to what one obtains in the simplest ratchet models. Interestingly, coupling two such ratchets by a spring [20,21] leads to a driving mechanism which is independent of the diffusion along the filament. Similarly, dimerization of monomeric kinesin results in a higher velocity and smaller diffusion coefficient of the filament-bound motors [17]. These results suggest that the dimerization of motors has two effects: It allows the motor to stay bound to the filament for many chemical cycles - in contrast to many (therefore unprocessive) monomeric motors - and in addition, it allows the motors to move by a more efficient mechanism which, in contrast to the motility of monomers, does not rely on diffusion along the filament.

\section{Random walks arising from many diffusional encounters with filaments}

\subsection{Motor walks in open compartments}

The fact that nanometer-sized motor molecules work in a noisy environment has another consequence: Even processive motors do not move along a filament forever, but unbind from it after a certain binding time (which corresponds to a typical walking distance), because the binding energy is finite and can be overcome by thermal fluctuations. For a single kinesin or myosin $\mathrm{V}$ motor, the binding times and walking distances are of the order of $1 \mathrm{~s}$ and $1 \mu \mathrm{m}$, respectively. Much longer walking distances can be obtained if several motors form a complex or if a cargo is transported by a larger number of motors. Note that the unbinding may also have a biological function by allowing the motors to diffuse around obstacles on the filament.

Unbound motors perform simple Brownian motion until they rebind to the same or another filament. On large time scales, the combination of active directed movements along filaments and non-directed Brownian motion leads to peculiar random walks, called motor walks in the following, which consist of alternating sequences of the two types of movements as sketched in Fig. 2 [22,23].

In order to determine the effective transport properties of these motor walks, we have studied several simple arrangements of filaments embedded in compartments of various geometries. A particularly simple but intriguing case consists of a single filament and a set of confining walls, which restrict the diffusion of unbound motors. In the simplest case, there are no confining walls and the unbound motors can diffuse freely in the full three-dimensional space (similar behavior is obtained for a half space geometry which is more easily accessible to experiments). By placing the filament in a quasi two-dimensional slab or in a cylindrical tube (geometries, which are also accessible to in vitro experiments), diffusion can be restricted along one or two dimensions perpendicular to the filament.

We have studied the motor walks by mapping them to random walks on a lattice [23]. A line of lattice sites represents the filament. Motors at these sites perform a biased random walk and move predominantly into one direction, which we choose to be the positive $x$ di- 
rection. In a discrete-time description, they move with a small probability $\epsilon / 2 d$ to each of the adjacent nonfilament sites and thus unbind from the filament. At the non-filament sites the motors perform simple symmetric random walks and move to each neighbor site with probability $1 / 2 d$ ( $d$ denotes the spatial dimension) and rebind to the filament with probability $\pi_{\mathrm{ad}}$ when they reach again a filament site. Confining walls are implemented as repulsive boundaries, at which all attempted movements towards the walls are rejected.

We have used scaling arguments, computer simulations, and exact solutions of the master equations to study the drift and diffusion behavior arising from the motor walks $[23,24,25,26]$. The motor walks exhibit anomalous drift behavior and strongly enhanced diffusion parallel to the filament due to the repeated binding and unbinding. At large times, motors move with an effective velocity given by $v_{\mathrm{b}} P_{\mathrm{b}}$, where $v_{\mathrm{b}}$ is the velocity of the bound motor and $P_{\mathrm{b}}$ is the probability that the motor is bound to the filament. In the tube geometry, $P_{\mathrm{b}}$ is time-independent at large times and given by the equilibrium of binding/unbinding and diffusion perpendicular to the filament. In the slab and half-space geometries as well as in two- and three-dimensional systems without confining walls, a steady state cannot be reached at any time, because motors can rebind to the filament after arbitrarily large excursions, and the typical size of these excursions, that contribute to the average behavior, increases with time $t$. Therefore, $P_{\mathrm{b}}$ and the effective velocity are time-dependent in these cases, namely $P_{\mathrm{b}}(t) \sim t^{-d_{\perp} / 2}$ for compartments with $d_{\perp}$ dimensions of unconfined diffusion (for $d$-dimensional systems without confining walls, we have $d_{\perp}=d-1$ ). The time-dependent effective velocity implies that the average displacement of the motors grows sublinearly. In the effectively two-dimensional slab geometry (as well as on a two-dimensional lattice without confining walls), the displacement behaves as $x(t) \sim \sqrt{t}$ at large times, and in the half space (or full three-dimensional space), it is given by $x(t) \sim \ln t$, see Fig. 3 .

In the following, we will give a simplified description which explains these features and relates them to known results from the theory of random walks.

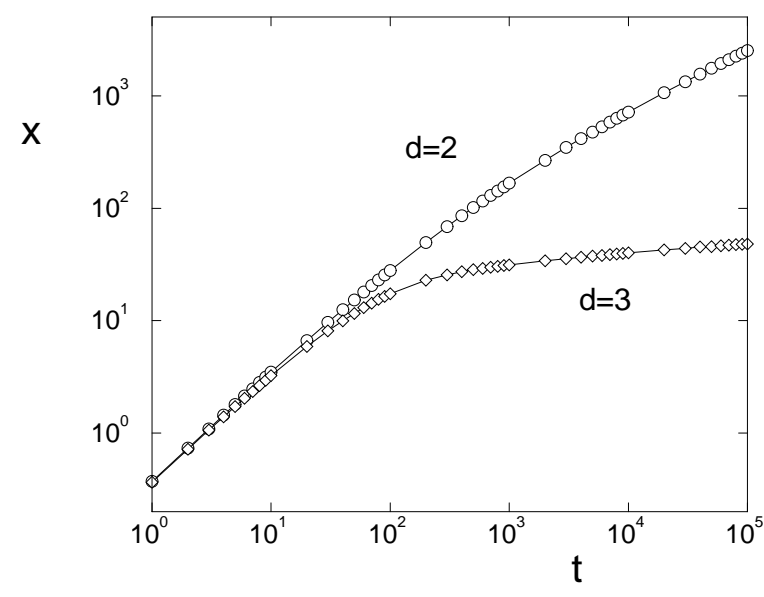

Fig. 3. Average displacement $x$ as a function of time $t$ for the motor walks as shown in Fig. 2 in two- and three-dimensional systems without confining walls. The displacement grows as $x \sim t^{1 / 2}$ and $x \sim \ln t$ for $d=2$ and $d=3$, respectively.

\subsection{Asymptotics and return to the filament}

For simplicity, we discuss the case of a single infinitely long filament embedded into $d$-dimensional space with $d=2$ or $d=3$. Motor particles binding to this filament walk along it with velocity $v_{\mathrm{b}}$ until they unbind. Unbinding occurs with a rate $\sim \epsilon$, so that the motors perform straight movements over the walking distance $\Delta x_{\mathrm{b}} \sim v_{\mathrm{b}} / \epsilon$. The diffusive excursions between two bound walks bring the motors back to the filament. (For simplicity, we take the sticking probability $\pi_{\mathrm{ad}}$ to be one. If $\pi_{\mathrm{ad}}<1$, the motor has to return to the filament $1 / \pi_{\mathrm{ad}}$ times before rebinding.) The distribution $\psi(\tau)$ of the excursion times $\tau$ is therefore given by the distribution of the return times of a random walker's return to a line in $d$ dimensions or, if we consider the projection into the plane perpendicular to the filament, of the return to the origin in $d-1$ dimensions. This is a classical problem in the theory of random walks which was solved by Polya in 1921 and has lead to the remarkable result that the return of a random walker to the origin is certain on one- and two-dimensional lattices, but not in three dimensions [27]. For the molecular motors, this implies that they will return to the filament with certainty.

If we are only interested in the movement parallel to the filament, we can consider the excursions away from the filament as periods of rest. In addition, for 
large times, the duration of an excursion is typically much longer than the time the motor is bound to the filament; therefore we can consider the walks along the filament as effectively instantaneous steps of size $x_{s} \sim$ $\Delta x_{\mathrm{b}} \sim v_{\mathrm{b}} / \epsilon$ (If $\pi_{\mathrm{ad}}<1$, the effective step size is $x_{s} \sim$ $v_{\mathrm{b}} \pi_{\mathrm{ad}} / \epsilon$. The motor walks are then described by continuous time random walks with the dwell time distribution $\psi(\tau)$ given by the distribution of the excursion times. For this type of random walks, solutions can be obtained using Fourier-Laplace transforms [28]. A short summary of this method is given in appendix $\mathrm{A}$. In that way, one finds that the Laplace transforms of the first two moments of the position of this random walker are given by

$x(s)=\frac{\left\langle x_{s}\right\rangle \psi(s)}{s[1-\psi(s)]}$

and

$x^{2}(s)=\frac{\left\langle x_{s}\right\rangle^{2} \psi(s)}{s[1-\psi(s)]}+\frac{2\left\langle x_{s}^{2}\right\rangle \psi^{2}(s)}{s[1-\psi(s)]^{2}}$

where $\psi(s)$ is the Laplace transform of the waiting time distribution $\psi(s) \equiv \int_{0}^{\infty} \mathrm{d} t \psi(t) e^{-s t}$. In general, $\left\langle x_{s}\right\rangle$ and $\left\langle x_{s}^{2}\right\rangle$ are the moments of the step size distribution; for the motor walks, they are given by the active walks along the filament and we take them to be given by $x_{s}=v_{\mathrm{b}} / \epsilon$ and $x_{s}^{2}$, respectively. From these relations, the average displacement and the dispersion of the motor walks for large times can be obtained by inverting the Laplace transform. In particular, these relations imply that the asymptotic displacement of the motors is given by the large-time (or small $s$ ) behavior of the distribution of return times to the origin in $d-1$ dimensions.

Normal drift behavior with $x(t) \sim t$ is obtained, as long as $\psi(\tau)$ has a finite mean value, $\bar{\tau}$, and thus $\psi(s) \approx$ $1-\bar{\tau} s$ for small $s$. If, however, $\psi(\tau)$ decays slower than $\sim \tau^{-2}$ at large $\tau$, the mean dwell time diverges (which implies a divergence of $[\psi(s)-1] / s$ for small $s$ ), and anomalous drift is obtained. The latter behavior occurs in our case (where the waiting times are given by return times to the filament) if the diffusion away from the filament is not restricted.

In the two-dimensional case, the return time distribution behaves as $\psi(\tau) \approx 1 /\left(2 \sqrt{\pi} \tau^{3 / 2}\right)$ for large $\tau$ or $\psi(s) \approx 1-\sqrt{s}$ for small $s$ as shown in appendix B. Inserting this into Eq. (1) and inverting the Laplace transform, we obtain $x(s) \approx \frac{x_{s}}{s^{3 / 2}}=\frac{v_{\mathrm{b}}}{\epsilon s^{3 / 2}}$

and

$x(t) \approx \frac{2 x_{s} \sqrt{t}}{\sqrt{\pi}}=\frac{2 v_{\mathrm{b}} \sqrt{t}}{\epsilon \sqrt{\pi}}$

for small $s$ and large $t$, respectively. Similarly, in the three-dimensional case, the return time distribution is $\psi(\tau) \approx 2 \pi /\left(3 \tau \ln ^{2} \tau\right)$ for large $\tau$ or $\psi(s) \approx 1-$ $2 \pi /\left(3 \ln s^{-1}\right)$ for small $s$, see again appendix B, which leads to

$x(t) \approx \frac{3 x_{s}}{2 \pi} \ln t=\frac{3 v_{\mathrm{b}}}{2 \pi \epsilon} \ln t$.

Likewise, we can obtain the dispersion $\Delta x^{2}(t)$ of the motors from the second moment of the distribution arising from the encounters with filaments. Eq. (2) leads to $\Delta x^{2}(t) \approx \frac{2(\pi-2)}{\pi}\left(v_{\mathrm{b}} / \epsilon\right)^{2} t+\frac{1}{2} t$ and $\Delta x^{2}(t) \approx$ $\frac{9}{4 \pi^{2}}\left(v_{\mathrm{b}} / \epsilon\right)^{2} \ln t+\frac{1}{3} t$ in two and three dimensions, respectively, where we have added the contribution due to the diffusion of unbound motors parallel to the filament. Note that the broadening of the distribution of motors due to the encounters with the filament is characterized by an anomalously high effective diffusion coefficient of the order of $\left(v_{\mathrm{b}} / \epsilon\right)^{2}$ in two dimensions, while in three dimensions, the leading term is the unbound diffusion, but with a large logarithmic correction, again of the order $\left(v_{\mathrm{b}} / \epsilon\right)^{2}$. These results agree with the corresponding asymptotic results from the exact solution of the full master equations $[24,25]$.

\section{Traffic phenomena in many-motor systems}

\subsection{Traffic jams and density patterns}

Finally, we consider systems with many interacting molecular motors. The simplest type of motor-motor interaction is simple exclusion which arises from the fact that a motor occupies a certain volume and, in particular, excludes other motors from the binding site of the filament to which it is bound as observed in decoration experiments, see, e.g., Ref. [29]. Exclusion is most important if motors accumulate in certain regions along the filaments, where it leads to the formation of molecular traffic jams. These interactions are easily incorporated into our lattice model by rejecting all movements to occupied lattice sites [23,30,31]. These 
models then represent new variants of exclusion processes or driven lattice gas models, where the active or driven movements are localized to the filaments. In driven exclusion processes, the state of the system depends crucially on the boundary conditions, since the boundaries determine the current through the system.

We have studied tube systems with a single filament located along the axis of a cylindrical tube and with several types of boundary conditions at the left and right tube ends $[23,30]$. We use the convention that the active movement along the filament is biased to the right. There are several possibilities to build such tube systems artificially such as micropipette glass tubes or liquid microchannels, but there are also several tubular compartments within cells for which these tube systems provide simple descriptions. The most prominent example for the latter is the axon of a nerve cell; another example is provided by the hyphae of fungi.

The simplest situation is given by periodic boundary conditions which can be solved exactly [30]. In this case, motor particles reaching the right end of the tube simply restart their movements at the left end. This leads to constant density profiles for both the bound and unbound motors in the stationary state. Diffusive currents in the radial direction vanish and binding to the filament is locally balanced by unbinding. The current of motors through the tube is given by $J=v_{\mathrm{b}} \rho_{\mathrm{b}}\left(1-\rho_{\mathrm{b}}\right)$ and the value of the bound density $\rho_{\mathrm{b}}$ is determined by the total number of motor particles in the tube. If the number of motors within the tube is increased beyond an optimal number (where $\rho_{\mathrm{b}}=1 / 2$ and $J=v_{\mathrm{b}} / 4$ ), the current through the tube decreases due to jamming of the motors.

In a closed tube, motors accumulate in front of the right end, and a diffusive current of unbound motors to the left balances the current along the filament in the stationary state $[23,31]$. If the number of motors in the tube is small, the motors are essentially localized at the right tube end. Upon increasing the number of motors within the tube, a jammed region at the right tube end builds up, separated from a low density region to its left by a rather sharp interface, which provides probably the simplest example for active pattern formation by molecular motors. The crowded domain spreads to the left at higher motor concentrations until the filament is uniformly covered by motors and rather crowded, see Fig. 4. Such density profiles have recently been observed for a kinesin-like motor in fungal hyphae (a)
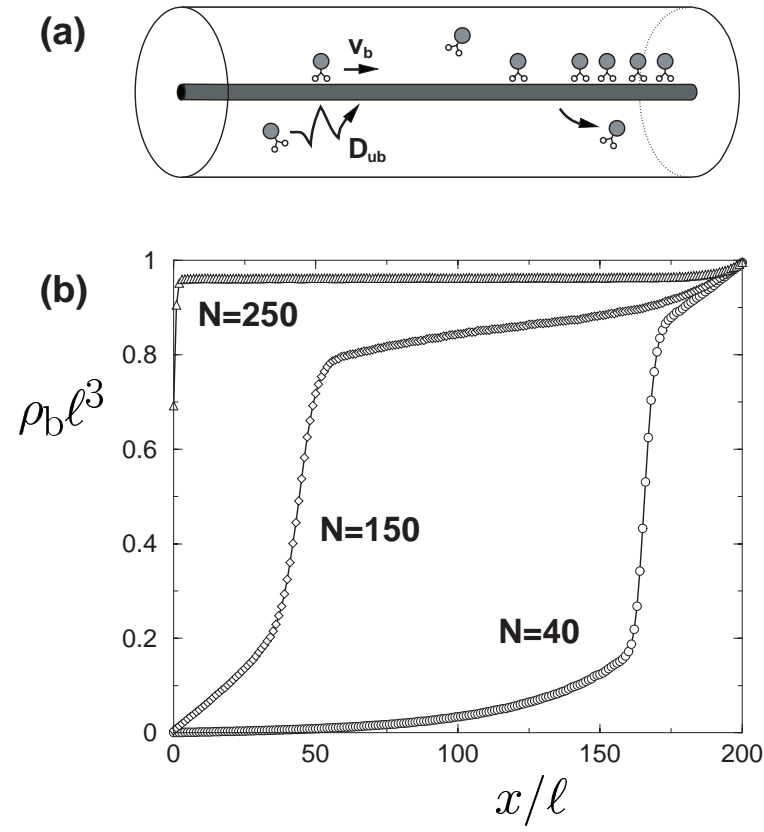

Fig. 4. (a) Motors moving in a closed tube system which contains one filament. The motors move with velocity $v_{\mathrm{b}}$ along the filament to the right and build up a motor traffic jam at the right end of the system. In the stationary state, the bound motor current is balanced by a current of unbound motors to the left arising from the diffusive movement of the unbound motors which is characterized by the diffusion coefficient $D_{\mathrm{ub}}$. (b) Profiles of the bound motor density as a function of the coordinate $x$ along the filament for various total numbers $N$ of motors within the tube. The jammed region becomes longer with increasing $N$.

[32]. Let us note that these density patterns exist due to continuous consumption of ATP. If the ATP concentration is not kept constant within the closed compartment, then after burning all ATP, the density pattern will finally become homogeneous.

Tubes with open boundaries which are coupled to reservoirs of motors at both ends, so that motors enter the tube at the left end and leave it at the right end, exhibit boundary-induced phase transitions [30,33]. There are three phases which are distinguished by the 'bottleneck' which determines the motor current through the tube. This 'bottleneck' can be the left boundary, the right boundary or the interior of the tube. These three cases corresponds to the low-density (LD), high-density (HD) and maximal-current (MC) phase as shown in Fig. 5 . If changing the motor densities in the reservoirs at the boundaries leads to a 


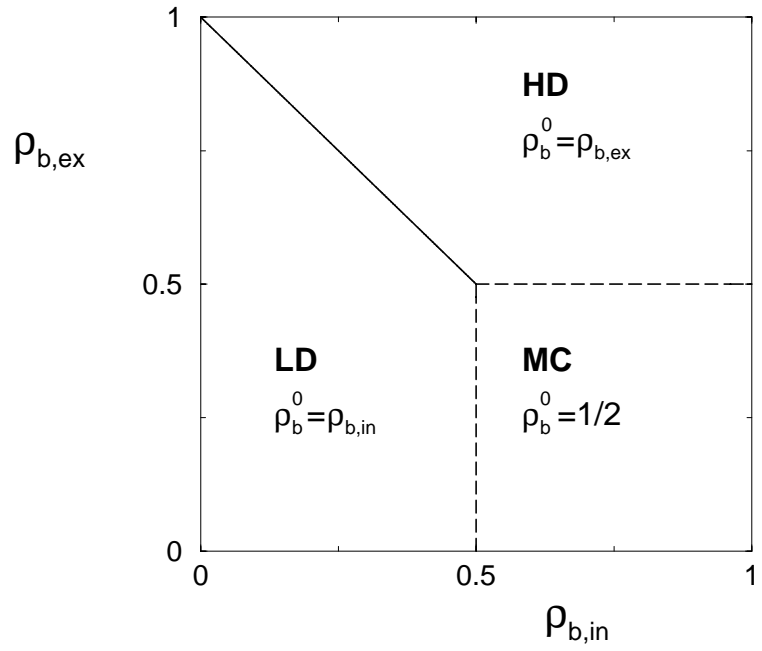

Fig. 5. Phase diagram for the motor traffic through an open tube which is coupled to motor reservoirs at the left and right end, characterized by bound motor densities $\rho_{\mathrm{b}, \text { in }}$ and $\rho_{\mathrm{b}, \text { ex }}$, respectively [30]. Three phases - a low density (LD) phase, a high density (HD) phase, and a maximal current (MC) phase - are distinguished by the value of the bulk density $\rho_{\mathrm{b}}^{0}$ in the interior of the tube. In the case shown here, bound and unbound motor densities satisfy radial equilibrium at the boundaries, for other choices of the boundary conditions, the transition lines are shifted.

change in the 'bottleneck' position, a phase transition occurs which can be either discontinuous (LD$\mathrm{HD}$ ) or continuous (LD-MC and HD-MC). These types of phases and transitions are known from the one-dimensional asymmetric simple exclusion process (ASEP) [34,35] which corresponds to the dynamics along the filament in our model without the binding and unbinding processes. The presence of the unbound state of the motors, however, increases the number of possible boundary conditions, and since the phase transitions are boundary-induced, the location of the transition lines within the phase diagram is quite sensitive to that choice. At present, it is difficult to see which of these boundary conditions will be the simplest to implement experimentally. Nevertheless, systems of molecular motors are promising candidates for the experimental observation of boundary-induced phase transitions.

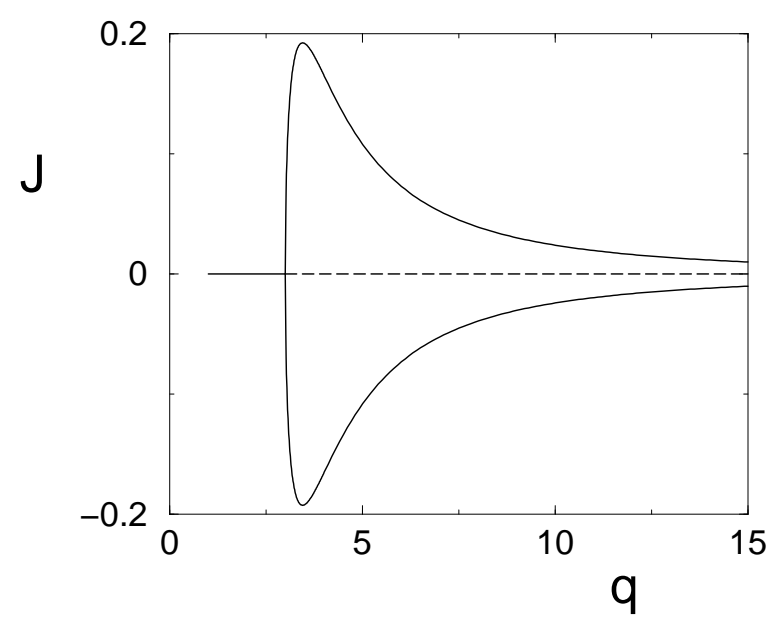

Fig. 6. Spontaneous symmetry breaking in systems with two motor species moving into opposite directions along the same filament: The total motor current $J$ is zero for weak interaction $q<q_{c}$, where the filament is equally populated by both motor species, but non-zero for sufficiently strong motor-motor interactions with $q>q_{c}$, where one motor species is essentially excluded from the filament [36]. For very strong interaction, the current decreases because the filament becomes more and more crowded.

\subsection{Phase transitions in two-way traffic}

Another type of phase transitions occurs in systems with two-way traffic of motors: While each motor moves either towards the plus- or towards the minusend of the corresponding filament, different types of motors move into opposite directions. We have studied systems with two species of motors moving into opposite directions along the same filament [36]. The interactions of these motors are described by a single interaction parameter $q$ by which the binding and unbinding rates are increased or reduced in order to enhance binding and reduce unbinding if a motor of the same type is present at a neighbor site and to enhance unbinding and reduce binding if a motor with opposite directionality is bound at a neighbor site. This type of interaction is suggested by decoration experiments. For equal concentrations of both motor species and for sufficiently strong interaction with $q>q_{c}$ (where $q_{c}$ is the critical value of the interaction parameter which depends on the overall motor concentration), there is spontaneous symmetry breaking, so that one motor species occupies the filament, while the other one is largely excluded from it, see Fig. 6 . 
If several filament are aligned in parallel and with the same orientation, this symmetry breaking leads to the spontaneous formation of traffic lanes for motor traffic with opposite directionality. Varying the relative concentration of the two motor species for $q>q_{c}$ leads to a discontinuous phase transition with hysteresis, similar to the transitions induced by changes of the external fields in magnetic systems. In contrast to the boundary-induced phase transitions discussed in the preceding section, these transitions do not depend on the boundaries, but are induced by the binding and unbinding dynamics. This implies that they are hardly affected by the choice of the boundary conditions. They depend, however, on the active movements of the motors, and are not found in an equilibrium situation with motor velocity $v_{\mathrm{b}}=0$, as applies, e.g., to systems without ATP.

\section{Summary}

In summary, molecular motors exhibit interesting movements on several length scales. Here we have addressed two of these length scales, namely the walks along filaments which consist typically of $\sim 100$ steps with a step size of the order of $10 \mathrm{~nm}$ and random walks which consist of many such walks along filament interrupted by periods of diffusion after unbinding from the filament. In addition, the presence of many motors leads to traffic phenomena such as traffic jams and traffic lanes, similar to the macroscopic traffic on streets. However, unbinding of motors from the filaments due to thermal fluctuations (which is a consequence of their microscopic size) plays an important role and can help to circumvent obstacles and to regulate the traffic.

\section{Appendix A. Continuous time random walks}

In this appendix, we summarize some results for random walks with a dwell time distribution $\psi(\tau)$ and a step distribution $\mathcal{P}\left(x_{s}\right)$ which are used in section 3 . We consider random walkers in one dimension which make the first step at time $t=0$ starting from the origin, $x=0$. The probability distribution $p(x, t)$ of such a random walk fulfills the recursion relation $[37,28]$ $p(x, t)=\sum_{n=0}^{\infty} p_{n}(x) \int_{0}^{t} \psi_{n}\left(t^{\prime}\right) \Psi\left(t-t^{\prime}\right) \mathrm{d} t^{\prime}$,

where $p_{n}(x)$ is the probability density that the walker is at position $x$ after the $n$ 'th step, $\psi_{n}(t)$ is the probability that the $n$ 'th step occurs at time $t$, and $\Psi(t) \equiv$ $\int_{t}^{\infty} \psi(\tau) \mathrm{d} \tau$ is the probability that no step occurred until time $t$. The initial conditions are $p_{0}(x)=\delta(x)$ and $\psi_{0}(t)=\delta(t)$. The solution of this recursion can be obtained using Fourier-Laplace transforms, which leads to

$p(q, s)=\frac{1-\psi(s)}{s[1-\mathcal{P}(q) \psi(s)]}$

for the Fourier-Laplace transform of the probability distribution $p(x, t)$ [37] with the Laplace transform of the waiting time distribution, $\psi(s) \equiv \int_{0}^{\infty} \mathrm{d} t \psi(t) e^{-s t}$ and the Fourier transform of the step distribution $\mathcal{P}(q) \equiv \int_{-\infty}^{\infty} \mathrm{d} x_{s} \mathcal{P}\left(x_{s}\right) e^{i q x_{s}} \approx 1+i\left\langle x_{s}\right\rangle q-\left\langle x_{s}^{2}\right\rangle q^{2} / 2$. The latter expansion is valid for small $q$, provided that the moments $\left\langle x_{s}^{n}\right\rangle$ of the step distribution $\mathcal{P}\left(x_{s}\right)$ with $n=1,2$ are finite. Using this expansion, we can derive expressions for the Laplace transforms of the moments of our random walk by expanding $p(q, s)$ as given in Eq. (A.2) in powers of $q$ [38] which leads to Eq. (1) from which the asymptotic behavior of the time-dependent moments can by obtained via the Tauberian theorems, see Ref. [28].

\section{Appendix B. Return to the origin}

In this appendix we sketch the derivation of the distribution of return times to the origin which determines the dwell time distribution $\psi$ used in section 3.2. We consider the probability $f(\mathbf{x}, t)$ that, at time $t$ the random walker is at position $\mathbf{x}$ for the first time provided it started at the origin at time $t=0 . f(\mathbf{0}, t)$ then determines the distribution of return times to the origin. These probabilities satisfy the recursion relation

$p(\mathbf{x}, t)=\sum_{\tau=1}^{t} f(\mathbf{x}, t-\tau) p(\mathbf{0}, \tau)+\delta_{t, 0} \delta_{\mathbf{x}, \mathbf{0}}$

which states that the walker is at site $\mathbf{x}$ at time $t$, if it had been there at any time $\tau<t$ and returned there in time $t-\tau$. The last term expresses the initial conditions. Using Fourier-Laplace transforms, one can derive an expression for the Laplace transform of $f(\mathbf{0}, t)$, 
the probability that the walker returns to the origin at time $t[27,28]$,

$f(\mathbf{0}, s)=\frac{1}{1+s}-\frac{1}{(1+s)^{2} J^{(d)}(s)}$

where the $J^{(d)}(s)$ are integrals over the momentum $\mathbf{q}$ which depend on the spatial dimensions. The same integrals have to be calculated for the exact solution of the motor walks [25].

In the one-dimensional case we have

$J^{(1)}(s)=\frac{1}{2 \pi} \int_{0}^{2 \pi} \frac{\mathrm{d} q}{s+\frac{1}{2}(1-\cos q)}=\frac{1}{\sqrt{s+s^{2}}}$

which leads to $f(\mathbf{0}, s) \approx 1-\sqrt{s}$ for small $s$ and

$f(\mathbf{0}, t) \approx \frac{1}{2 \sqrt{\pi} t^{3 / 2}}$

for large $t$. In the two-dimensional case the corresponding integral is given by

$$
\begin{aligned}
J^{(2)}(s) & =\frac{1}{(2 \pi)^{2}} \int_{0}^{2 \pi} \int_{0}^{2 \pi} \frac{\mathrm{d} q_{1} \mathrm{~d} q_{2}}{s+\frac{1}{3}\left(2-\cos q_{1}-\cos q_{2}\right)} \\
& =\frac{3 \sqrt{m}}{\pi} K(m),
\end{aligned}
$$

where $K(m)$ is a complete elliptic integral of the first kind and $m \equiv 4 /(2+3 s)^{2}$. Since $K(m)$ behaves as $K(m) \approx \frac{1}{2} \ln \frac{16}{1-m}$ for $m$ close to one [39], the return time distribution is asymptotically given by $f(\mathbf{0}, s) \approx$ $1-2 \pi /\left(3 \ln s^{-1}\right)$ for small $s$ or

$f(\mathbf{0}, t) \approx \frac{2 \pi}{3 t \ln ^{2} t}$

for large $t$.

The dwell time distribution $\psi(\tau)$ used in section 3.2 for the case of a filament within a $d$-dimensional lattice is given by $\psi(\tau)=f(\mathbf{0}, t=\tau)$, where the expression on the right hand side has to be taken in the $d_{\perp}=d-1$ dimensions.

\section{References}

[1] J. Howard, Mechanics of Motor Proteins and the Cytoskeleton, Sinauer Associates, Sunderland (Mass.), 2001.
[2] M. Schliwa (Editor), Molecular motors, Wiley-VCH, Weinheim, 2003

[3] H. Hess, V. Vogel, Rev. Mol. Biotechnology 82 (2001) 67-85.

[4] K. J. Böhm, E. Unger, in: H. S. Nalwa (Editor), Encyclopedia of Nanoscience and Nanotechnology Vol.4, American Scientific Publishers, Stevenson Ranch, 2004, pp. 345-357.

[5] R. Lipowsky, S. Klumpp, Physica A (in press).

[6] K. Svoboda, C. F. Schmidt, B. J. Schnapp, S. M. Block, Nature 365 (1993) 721-727.

[7] C. L. Asbury, A. N. Fehr, S. M. Block, Science 302 (2003) 2130-2134.

[8] A. Yildiz, M. Tomishige, R. D. Vale, P. R. Selvin, Science 303 (2004) 676-678.

[9] A. Yildiz, J. N. Forkey, S. A. McKinney, T. Ha, Y. E. Goldman, P. R. Selvin, Science 300 (2003) 2061-2065.

[10] K. Visscher, M. J. Schnitzer, S. M. Block, Nature 400 (1999) 184-189.

[11] F. Jülicher, A. Ajdari, J. Prost, Rev. Mod. Phys. 69 (1997) 1269-1281.

[12] R. Lipowsky, in: J. Freund, T. Pöschel (Eds.), Stochastic processes in physics, chemistry and biology, Springer, Berlin, 2000, pp. 21-31.

[13] R. D. Astumian, P. Hänggi, Physics Today 55 (11) (2002) 33-39.

[14] R. Lipowsky, Phys. Rev. Lett. 85 (2000) 4401-4405.

[15] R. Lipowsky, N. Jaster, J. Stat. Phys. 110 (2003) 11411167.

[16] Y. Okada, N. Hirokawa, Science 283 (1999) 1152-1157.

[17] M. Tomishige, D. R. Klopfenstein, R. D. Vale, Science 297 (2002) 2263-2267.

[18] Y. Okada, H. Higuchi, N. Hirokawa, Nature 424 (2003) $574-577$.

[19] M. Schliwa, G. Woehlke, Nature 422 (2003) 759-765.

[20] A. Ajdari, J. Phys. I France 4 (1994) 1577-1582.

[21] S. Klumpp, A. Mielke, C. Wald, Phys. Rev. E 63 (2001) 031914.

[22] A. Ajdari, Europhys. Lett. 31 (1995) 69-74.

[23] R. Lipowsky, S. Klumpp, T. M. Nieuwenhuizen, Phys. Rev. Lett. 87 (2001) 108101.

[24] T. M. Nieuwenhuizen, S. Klumpp, R. Lipowsky, Europhys. Lett. 58 (2002) 468-474.

[25] T. M. Nieuwenhuizen, S. Klumpp, R. Lipowsky, Phys. Rev. E 69 (2004) 061911.

[26] T. M. Nieuwenhuizen, S. Klumpp, R. Lipowsky, Physica A (in press). 
[27] G. Polya, Math. Ann. 84 (1921) 149-160.

[28] G. Weiss, Aspects and Applications of the Random Walk, North-Holland, Amsterdam, 1994.

[29] Y.-H. Song, E. Mandelkow, Proc. Natl. Acad. Sci. USA 90 (1993) 1671-1675.

[30] S. Klumpp, R. Lipowsky, J. Stat. Phys. 113 (2003) 233-268.

[31] S. Klumpp, T. M. Nieuwenhuizen, R. Lipowsky, Biophys. J. (in press).

[32] S. Konzack, P. E. Rischitor, C. Enke, R. Fischer, Mol. Biol. Cell 16 (2005) 497-506.

[33] S. Klumpp, R. Lipowsky, Phys. Rev. E 70 (2004) 066104.

[34] J. Krug, Phys. Rev. Lett. 67 (1991) 1882-1885.

[35] A. B. Kolomeisky, G. M. Schütz, E. B. Kolomeisky, J. P. Straley, J. Phys. A: Math. Gen. 31 (1998) 69116919.

[36] S. Klumpp, R. Lipowsky, Europhys. Lett. 66 (2004) 90-96.

[37] E. Montroll, G. Weiss, J. Math. Phys. 6 (1965) 167 181.

[38] M. Shlesinger, J. Stat. Phys. 10 (1974) 421-434.

[39] M. Abramowitz, I. Stegun (Eds.), Pocketbook of Mathematical Functions, Harri Deutsch, Thun and Frankfurt, 1984. 